\title{
Enjeux d'une recherche collaborative menée avec trois enseignantes atikamekws à l'éducation préscolaire
}

Auteure(s)

Elisabeth Jacob, Université du Québec à Chicoutimi elisabeth1 jacob@uqac.ca

Annie Charron, Université du Québec à Montréal charron.annie@uqam.ca 


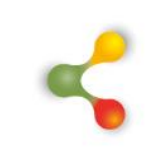

\section{REVUE HYBRIDE DE L'ÉDUCATION}

\section{Résumé}

L'intérêt de documenter les rôles d'enseignantes atikamekws à l'éducation préscolaire en contexte de jeu symbolique pour favoriser l'émergence de l'écrit provient d'une double préoccupation ; d'abord, un besoin provenant de trois enseignantes atikamekws qui souhaitaient avoir des outils pour favoriser le langage oral et écrit des enfants, ensuite celui de questionner scientifiquement les rôles d'enseignantes. Pour répondre à cette double préoccupation, une recherche collaborative a été menée dans trois classes d'éducation préscolaire en contexte autochtone. Cet article propose une réflexion sur le développement professionnel des enseignantes autochtones et la collaboration des acteurs permettant le bon déroulement de ce projet de recherche.

Mots-clés : éducation préscolaire; jeu symbolique; émergence de l'écrit; rôles d'enseignantes; recherche collaborative; développement professionnel 


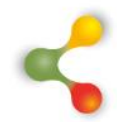

\section{REVUE HYBRIDE DE L'ÉDUCATION}

\section{Introduction}

Mes expériences de pratique comme consultante en petite enfance m'ont amenée à travailler dans les communautés autochtones à la suite d'un projet de recherche de maitrise réalisé avec des parents autochtones. Mon intérêt de réaliser un second projet de recherche cadrant dans les exigences d'un doctorat en éducation a vu le jour en 2011; en tant que consultante et jeune chercheuse, ce projet devait avoir des retombées dans la communauté autochtone. Les membres de la communauté atikamekw concernée ainsi que les enseignantes autochtones avec qui je collaborais m'ont fait confiance pour le réaliser. Dès 2012, plusieurs discussions se sont déroulées avec elles pour que le projet de recherche puisse répondre à leurs besoins, notamment celui de les soutenir dans leur pratique. Déjà passionnée par le sujet du jeu symbolique, celui de l'émergence de l'écrit est venu s'y greffer. C'est donc à partir de ces discussions et des besoins soulevés par ces enseignantes autochtones que la problématique de recherche s'est dessinée.

Ce texte apporte une réflexion liée à quelques enjeux d'une recherche collaborative mise en place pour un projet de recherche doctoral (2012-2017) dans une communauté autochtone du Québec. Pour aborder cette réflexion, la problématique, le cadre théorique, la méthodologie et quelques résultats de la recherche collaborative seront exposés. Ces résultats ouvriront une discussion, dans un premier temps, sur le développement professionnel des enseignantes autochtones à l'éducation préscolaire et, dans un deuxième temps, sur l'implication et la collaboration des acteurs concernés qui ont contribué au bon déroulement de la recherche.

\section{Problématique}

L'éducation des enfants autochtones a connu plusieurs changements au cours des dernières années. La période des pensionnats de 1840 à 1950 a eu des conséquences pour les enfants, dont la perte d'identité, de leur culture ainsi que celle de la langue autochtone. Aujourd'hui, l'éducation des enfants qui vivent dans les communautés autochtones du Québec est assurée par les services éducatifs dans chacune de celles-ci. De nombreuses communautés ont fait le choix d'instruire les enfants dans la langue autochtone pour des raisons d'ordres sociolinguistique, pédagogique et professionnel, notamment en contextes éducatifs de la petite enfance, dont l'éducation préscolaire (de la Sablonnière, Usborne et Taylor 2011; Hot, 2010; Norris, 2015).

Dans les communautés autochtones du Québec, on dénombre principalement trois contextes éducatifs de la petite enfance : les centres de la petite enfance (CPE), le programme d'aide préscolaire aux Premières Nations et l'éducation préscolaire (maternelle 4 ans et maternelle 5 ans) (Jacob, Charron et da Silveira, 2015a). L'éducation préscolaire marque un 


\section{$\&$}

\section{REVUE HYBRIDE DE L'ÉDUCATION}

passage important dans la vie de ces jeunes enfants puisqu'ils font leur entrée à l'école parfois dès l'âge de 4 ans. Ce contexte d'éducation crée parfois un flou dans les attentes des enseignantes qui se répercutent dans leurs pratiques éducatives (Larivée, 2011). Ces pratiques sont diversifiées et certaines peuvent être plus scolarisantes, dont celles liées à la découverte de l'écrit, d'où l'importance pour les chercheurs de s'y intéresser (Lynch, 2015a). De plus, l'éducation préscolaire se situe à michemin entre les attentes du programme éducatif des centres de la petite enfance, où les thèmes du développement global et du jeu constituent des principes de base, et du programme scolaire de la première année au primaire. Pourtant, le développement global et le jeu constituent aussi deux principes de base des programmes d'éducation préscolaire 4 et 5 ans (ministère de l'Éducation du Québec [MEQ], 2001; ministère de l'Éducation et de l'Enseignement supérieur [MEES], 2017).

Dans la classe d'éducation préscolaire, la mise en place d'activités significatives en émergence de l'écrit par les enseignantes dans des contextes d'écriture variés, ludiques et spontanés, notamment le contexte du jeu, permettra à l'enfant de se développer. Les composantes de l'émergence de l'écrit sont le langage oral, la clarté cognitive, la connaissance des lettres, la conscience phonologique et le principe alphabétique (Giasson, 2011). L'enfant développe des compétences en émergence de l'écrit qui s'acquièrent lorsqu'il interagit avec un adulte et discute à propos de l'écrit (Thériault et Lavoie, 2004). L'enfant autochtone d'âge préscolaire a déjà acquis un certain bagage en émergence de l'écrit variant selon son environnement familial (Battle, 2009).

Plusieurs chercheurs recommandent que les activités en émergence de l'écrit proposées à l'éducation préscolaire aux enfants autochtones soient réalisées dans leur langue maternelle afin de consolider cette langue, mais aussi pour développer leur identité culturelle et faciliter le passage vers l'apprentissage de la langue seconde (Ball, 2010 ; Francis et Reyhner, 2002 ; Jacob et al., 2015a). Dans les classes non autochtones, un environnement propice à la lecture et à l'écriture comprenant, entre autres, des livres variés (p. ex. : contes, documentaires albums sans texte) vont permettre aux enfants de développer des compétences en émergence de l'écrit (Montésinos-Gelet, Dupin de Saint-André et Bourdeau, 2015). Le peu de livres écrits en langue autochtone et destinés aux enfants d'âge préscolaire (Ball, 2007, 2009, 2010 ; Conseil en Éducation des Premières Nations [CPEN], 2012 ; Institut culturel éducatif montagnais [ICEM], 2007 ; Jacob, 2012) est une réalité à laquelle les enseignantes autochtones sont confrontées (Jacob et al., 2015b). Auprès des enfants autochtones, des pratiques éducatives holistiques, communautaires et intégrant la culture et les savoirs autochtones (p. ex. : contes, légendes, respect des traditions orales et du territoire) sont à préconiser pour soutenir leur développement (Lavoie, 2016 ; Toulouse, 2008). 


\section{$\&$ \\ REVUE HYBRIDE DE L’ÉDUCATION}

L'une de ces pratiques est notamment d'utiliser le jeu comme contexte pour permettre aux enfants de découvrir l'écrit. Les enfants autochtones s'adonnent au jeu en centre de la petite enfance, en classe d'éducation préscolaire et à la maison, que ce soit avec leurs parents, leurs grands-parents et leur fratrie (Jacob, 2013, 2015a; Peterson, Madsen, San Miguel et Young, 2018). D'ailleurs, les parents, les éducateurs et les enseignants autochtones considèrent le jeu comme étant important pour le développement cognitif, langagier et socioaffectif des enfants (Gillis, 1991; Jacob, 2012; Peterson et al., 2018). Entre 4 et 6 ans, les enfants vont davantage s'adonner au jeu symbolique plutôt qu'à une autre forme de jeu (Bodovra et Leong, 2012). En jouant, les enfants autochtones s'approprient le langage pour plusieurs fonctions, notamment pour apprendre, pour s'imaginer et pour exprimer un événement réel de leur quotidien (Eisazadeh, Rajendram, Portier et Peterson, 2017). De plus, dans ce contexte, ils élaborent des scénarios de jeu autour d'activités culturelles et significatives pour eux (ex. : chasse, pêche, maison) leur permettant de construire un système de signifiants de leur culture autochtone (Eisazadeh et al., 2017). D'ailleurs, à travers le jeu, les enfants construisent leur compréhension de leur culture et s'appréhendent le monde adulte, autant dans les espaces publics, les classes et d'autres réseaux sociaux (Juster et Leichter-Saxby, 2014).

La valeur du jeu en classe d'éducation préscolaire est encore parfois méconnue par certaines enseignantes (Boudreau et Charron, 2014 ; Lynch, 2015b). Une conception erronée à l'égard du jeu, un manque de connaissances sur le jeu symbolique et l'émergence de l'écrit ainsi qu'un manque de temps pour enrichir les coins de jeu justifient un besoin de formation auprès des enseignantes à l'éducation préscolaire du Québec (Boudreau et Charron, 2014). Les enseignantes autochtones font face à ces mêmes défis (Jacob, Charron et da Silveira, 2015b). De plus, certaines, sans formation en éducation, sont embauchées dans les classes d'éducation préscolaire, ce qui permet de combler le manque de ressources dans les écoles de ces communautés autochtones (CEPN, 2002 ; Commission de l'éducation, 2007). Parce qu'elles maitrisent la langue autochtone à l'oral, conséquemment, on peut supposer qu'elles soutiennent les enfants dans leur développement langagier. Toutefois, cette situation peut engendrer des lacunes dans l'organisation et l'aménagement d'une classe d'éducation préscolaire ainsi que dans les approches pédagogiques proposées aux enfants. L'importance de documenter les rôles d'enseignantes autochtones en contexte de jeu symbolique pour comprendre comment elles soutiennent les enfants en émergence de l'écrit demeure un besoin scientifique à explorer (Roskos et Neuman, 1993; Saracho, 2002), parallèlement à leur besoin d'être formées au jeu symbolique et à l'émergence de l'écrit (Jacob et al., 2015b).

Simultanément à ce constat scientifique, trois enseignantes autochtones d'une même communauté ont exprimé un besoin d'être accompagnées dans leur pratique pour mieux soutenir le développement du langage oral et du langage écrit des enfants autochtones. Le projet de 


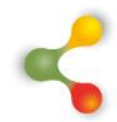

\section{REVUE HYBRIDE DE L'ÉDUCATION}

recherche s'est alors développé à partir d'une préoccupation conjointe : les besoins d'une chercheuse inscrite au doctorat en éducation souhaitant documenter un objet de recherche et s'intéressant au jeu et les besoins de praticiennes souhaitant mettre en place des activités significatives en émergence de l'écrit, recevoir de la formation et bénéficier de matériel de jeu et de mots écrits en atikamekw pour enrichir leurs coins.

L'objectif principal de cette recherche est de : 1) analyser les rôles d'enseignantes atikamekws à l'éducation préscolaire en contexte de jeu symbolique pour favoriser l'émergence de l'écrit.

\section{Quelques balises du cadre théorique}

Pour cet article, la perspective historico-culturelle sur le jeu sera décrite ainsi que le concept des rôles d'enseignantes. Le paradigme de recherche autochtone, un cadre théorique sur lequel prennent appui les fondements de cette recherche, sera présenté. Ces concepts sont davantage définis dans la thèse (Jacob, 2017).

\section{Perspective historico-culturelle du jeu}

Dans la théorie historico-culturelle de Vygotski (1967), la pensée et le langage sont d'origine socioculturelle et s'acquièrent grâce aux relations entre les personnes. Quant au jeu, il est essentiel et est la source principale pour le développement cognitif, affectif, langagier et social de l'enfant. Dans cette perspective, le jeu est défini selon trois critères: une situation imaginaire créée par l'enfant, l'adoption et l'interprétation de rôles et l'application de règles correspondant aux rôles de l'enfant dans le jeu (Vygotski, 1967). Une fonction symbolique est attribuée au jeu des enfants d'âge préscolaire et ce dernier facilite la séparation de la pensée des actions et des objets. Dans le jeu, l'enfant voit un objet, mais agit différemment selon sa perception. Ses actions sont conçues selon ses propres idées ou son imagination. Cette séparation amène le développement des idées plus abstraites, dont l'apprentissage de l'écriture (Vygotski, 1967). Les gestes de l'enfant associés à la fonction du jeu symbolique représentent une forme de langage.

S'inspirant des travaux de Vygotski, d'autres auteurs soulignent le lien entre le jeu symbolique et l'émergence de l'écrit (Hall, 2009; Jacob et al., 2015a; Pellegrini, 1980; Sawyer et Dezutter, 2009). Les transformations symboliques (p. ex., une chaise devient une motoneige dans un jeu), le langage oral, le métajeu (p. ex., l'enfant prend conscience de son jeu et communique des informations à propos de son jeu à ses partenaires) et les compétences narratives (p. ex., la manière dont l'enfant construit un scénario de jeu) mis en forme dans le jeu symbolique aideront l'enfant à se représenter les symboles de l'écriture, à découvrir la forme du langage écrit et à comprendre une histoire (orale ou écrite). 


\section{$\&$ \\ REVUE HYBRIDE DE L'ÉDUCATION}

\section{Rôles des enseignantes}

L'enseignante en éducation préscolaire, dans une pédagogie du jeu, a notamment comme mandat de préparer l'environnement de la classe, d'organiser et d'aménager le matériel, d'être une partenaire de jeu, contribuant ainsi à augmenter le niveau de jeu des enfants, leurs interactions sociales et leurs habiletés cognitives (de Haan, 2005; Gmitrovà et Gmitrov, 2003). Quelques écrits scientifiques portent sur les rôles des enseignantes, c'est-à-dire la manière dont elles agissent en contexte du jeu symbolique pour favoriser l'émergence de l'écrit (Bodovra et Leong, 2012; Neuman et Roskos, 1993; Roskos et Neuman, 1993; Saracho, 2002). Sept rôles y sont documentés en fonction du fait que l'enseignante adopte une posture de joueuse (p. ex. : (les enfants amorcent le jeu et l'enseignante suit leurs idées), de leader (p. ex. : l'enseignante structure le jeu) ou de spectatrice (p. ex. l'enseignante observe le jeu) (Bodovra et Leong, 2012 ; Neuman et Roskos, 1993 ; Roskos et Neuman, 1993 ; Saracho, 2002).

Prenons l'exemple du thème du restaurant pour décrire les sept rôles. L'enseignante joue un rôle d' "animatrice" Iorsqu'elle soutient l'enfant dans l'activité en émergence de l'écrit à partir de ses connaissances initiales (p. ex., l'enfant écrit un B pour désigner le mot banane, elle l'aide à écrire d'autres lettres à partir des sons qu'il entend). Elle joue le rôle de "conteuse " lorsqu'elle lit ou raconte une histoire ( $p$. ex., livres, histoire à l'oral) dans le jeu (p. ex., un client du restaurant lit un journal). Elle est «évaluatrice», lorsqu'elle questionne les enfants (p. ex., pourquoi le serveur a-t-il besoin d'un bloc de notes pour écrire?). Elle est " guide " lorsqu'elle réalise des interventions comme d'aider les enfants à planifier le jeu, donner des idées de thèmes aux enfants (p. ex., l'épicerie, le restaurant, la construction, etc.), choisir des accessoires et du matériel appropriés, nommer les mots affichés, les signes et leurs fonctions (p. ex., elle désigne aux enfants que le restaurant est ouvert, en pointant l'affiche du restaurant). Elle est "informatrice», lorsqu'elle explique des mots de vocabulaire nouveau dans le jeu (p. ex., la soupe au fenouil est dans le menu du midi. Le fenouil est un légume vert et croquant. II a un goût anisé, comme le goût d'une réglisse noire). Elle est "médiatrice» lorsqu'elle surveille l'évolution du jeu pour le rendre vers un jeu symbolique mature (Bodovra et Leong, 2012), lie des thèmes de jeu ( $p$. ex., le thème du restaurant et celui de l'hôpital), encadre les enfants qui ont besoin d'aide et aide les enfants à résoudre des conflits. Elle est "décideuse", puisqu'en contexte de jeu symbolique, elle prend des décisions spontanées pour suivre les intérêts de jeu de l'enfant et l'activité en émergence de l'écrit et elle prend des décisions réfléchies à l'égard de la planification.

Ce portrait sur les rôles des enseignantes est incomplet puisqu'il ne permet pas de les situer dans un contexte culturel spécifique, dont celui des classes d'éducation préscolaire en milieu autochtone. Les rôles identifiés dans les écrits proviennent principalement de recherches 


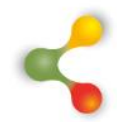

\section{REVUE HYBRIDE DE L'ÉDUCATION}

réalisées aux États-Unis (Neuman et Roskos, 1993 ; Roskos et Neuman, 1993; Saracho, 2002), et ce, auprès d'enseignantes de maternelle déjà formées sur le jeu symbolique et l'émergence de l'écrit.

\section{Paradigme de recherche autochtone}

La communauté scientifique reconnait depuis une trentaine d'années le paradigme de recherche autochtone soulignant l'importance de reconnaitre le savoir autochtone (Smith, 2012). Dans ce paradigme, la réalité est multiple; elle n'est jamais un objet, mais plutôt un ensemble de relations entre les éléments du monde autochtone, notamment la culture, le temps, le langage, l'histoire et la spiritualité (Wilson, 2003, 2008). Les connaissances se construisent grâce aux composantes de l'environnement, par exemple, les relations entre le chercheur et les participants de l'étude, l'univers, les animaux, les plantes et l'environnement au complet. Le respect, la réciprocité et la responsabilité font partie des principes axiologiques de ce paradigme (Smith, 2012; Weber-Pillwax, 2001; Wilson 2008). Enfin, selon l'ensemble des chercheurs, une méthodologie dont le chercheur respecte les principes éthiques de la recherche avec des Autochtones et maintient une qualité de la relation avec les participants (avant, pendant et après la recherche) pourra s'inscrire dans ce paradigme (Battiste, 2013 ; Chilisa, 2012 ; Smith, 2012 ; Weber-Pillwax, 2011). Tout chercheur allochtone peut donc entrer dans un "processus d'indigénisation» (Smith, 2012, p. 147, traduction libre) et conduire des recherches utilisant ce paradigme s'il partage les valeurs et la philosophie qui le sous-tendent (Chilisa, 2012 ; Smith, 2012 ; Wilson, 2013). Le chercheur doit reconnaitre le savoir des participants tiré du territoire, du langage, des histoires et du monde autochtones. Dans le projet de recherche concerné, la construction d'un nouveau savoir portant sur les rôles d'enseignantes atikamekws n'est possible qu'en agissant en étroite collaboration avec ces praticiennes et en faisant émerger leurs connaissances.

\section{Méthodologie}

Dans cette section, la recherche collaborative, le recrutement et les caractéristiques des participantes ainsi que les outils de collecte de données sont exposés.

\section{La recherche collaborative}

Une recherche collaborative, ancrée dans un paradigme de recherche autochtone, a été menée avec trois enseignantes. Le modèle développé par Desgagné (2001) a été choisi pour répondre aux objectifs de cette recherche. Le choix d'utiliser cette approche méthodologique, plutôt qu'une autre forme de recherche participative (p. ex., rechercheaction, recherche-formation ou recherche-intervention) réside dans le 


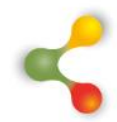

\section{REVUE HYBRIDE DE L'ÉDUCATION}

potentiel de faire émerger un savoir autochtone lié aux rôles d'enseignantes au regard de leur culture. Même si un besoin de formation est soulevé dans la problématique, les participantes possèdent une expertise liée à leur culture et à leur contexte de classe. Leur expertise, couplée à celle de la chercheuse, devient un «maillage » (Proulx, 2013) pour faire avancer les connaissances sur les rôles des enseignantes en contexte d'éducation préscolaire autochtone.

Le projet s'est effectué en trois étapes respectant ainsi le modèle proposé par Desgagné (Desgagné, 1997, 2001; Desgagné, Bednarz, Lebuis et al., 2001), à savoir la cosituation, la coopération et la coproduction.

Dans l'étape de cosituation, la chercheuse et les participantes ont décidé le thème de la recherche ainsi que leur besoin de formation. Cette démarche s'est échelonnée de 2012 à 2014. L'appui de la direction des services éducatifs, de la direction de l'école, mais surtout l'accord des membres du Conseil de bande ${ }^{1}$, ont été nécessaires. À l'automne 2014, des entrevues semi-dirigées auprès des trois enseignantes ont été menées pour comprendre l'importance qu'elles accordent au jeu dans leur classe, l'aménagement de leur coin de jeu symbolique ainsi que les activités en émergence de l'écrit proposées aux enfants de leur classe. Ce portrait nous permettait de mieux planifier la prochaine étape de la recherche.

À l'étape de coopération, une activité réflexive a été conduite avec les praticiennes afin qu'elles puissent avoir un espace pour questionner et expliciter leur pratique (Desgagné, 2001). Cette étape s'est déroulée en quatre boucles de planification, de mise en pratique dans la classe et d'analyse réflexive. Cette étape s'est échelonnée de janvier à mai 2015. Une rencontre de groupe, accompagnée par la chercheuse, permettait aux enseignantes de planifier un coin de jeu enrichi de matériels écrits (ex. : mots affichés, matériel de jeu avec des lettres de l'alphabet, livres, revues, crayons et feuilles) et de réfléchir aux stratégies et aux actions qu'elles peuvent faire pour favoriser l'émergence de l'écrit dans ce coin. L'aménagement du coin de jeu dans la classe précédait la mise en pratique. Durant celle-ci, les enseignantes guidaient les enfants dans le jeu en fonction des intérêts et des besoins de ces derniers. Après la mise en pratique dans la classe, un retour réflexif était réalisé avec les enseignantes pour qu'elles expliquent leur pratique. Quatre coins de jeu enrichis de matériels écrits portant sur quatre thèmes ont été conjointement planifiés, élaborés et mis en place dans la classe: le restaurant, l'hôpital, la

\footnotetext{
${ }^{1}$ Le Conseil de bande est l'assemblée des élus gouvernant chaque communauté. Il agit à titre de représentant auprès des gouvernements fédéral et provinciaux. II joue un rôle politique et administratif au sein de la communauté et assure la prestation des services (p. ex. : éducation, santé, habitations) pour les membres de la communauté.
} 


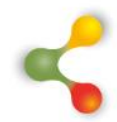

\section{REVUE HYBRIDE DE L'ÉDUCATION}

construction et la chasse. Par exemple, pour le coin de la chasse, des revues, des livres et des mots écrits, tel que tente, ours, outils, chasse, ont été ajoutés dans le coin de jeu. Du papier et des crayons étaient également mis à la disposition des enfants. Pour chacun des coins, la langue privilégiée à l'oral et à l'écrit était l'atikamekw, la langue autochtone parlée dans la communauté concernée.

La dernière étape, celle de coproduction consistait à la publication des résultats de la recherche et de ses retombées qui sont en termes de développement professionnel, mais aussi de la production de matériel, comme ce fut le cas pour les aménagements des coins de jeu symbolique enrichis de matériels écrits. Les enseignantes ont conservé le matériel de jeu acheté et le matériel écrit conçu durant le projet. Les résultats continus d'être diffusés sous différentes formes (p. ex., présentations, communications et publications, tant professionnelles que scientifiques) et à différents publics cibles (p. ex. : participantes, praticiens, étudiants, chercheurs).

\section{Participantes}

Une communauté autochtone au Québec a été sélectionnée pour participer à cette présente étude, reposant notamment sur le critère de la qualité de la relation (Kovach, 2009 ; Wilson, 2013). Des membres de cette communauté avaient déjà participé à un projet de recherche avec la chercheuse (Jacob, 2012). De plus, comme la chercheuse était consultante pour les services éducatifs de cette communauté, cela a permis de "saisir de l'intérieur » les besoins des participantes. La présence prolongée de la chercheuse dans le milieu et les rapports harmonieux avec plusieurs membres rejoignent des principes du paradigme de recherche autochtone (Battiste, 2013; Smith, 2012).

Trois enseignantes atikamekws ont participé au projet. Elles ont des formations et des expériences de travail diversifiées, mais toutes enseignent à l'éducation préscolaire dans une école située dans une communauté atikamekw. Une enseignante a obtenu son diplôme de baccalauréat en éducation préscolaire et en enseignement primaire et possède six années d'expérience en maternelle 5 ans (enseignante $B$ ) ; une enseignante détient une attestation d'études collégiales en petite enfance et possède six années d'expérience en maternelle 4 ans (enseignante A); et la troisième enseignante ne détient pas de diplôme postsecondaire et n'a aucune expérience comme enseignante à l'éducation préscolaire au moment de commencer la mise en œuvre du projet (enseignante $\mathrm{C}$ ).

Tout au long de cette recherche collaborative, le critère de « double vraisemblance », évoqué par Dubet (1994), a été respecté (Bednarz, 2013; Desgagné, 2001). Cela consistait à trouver un équilibre entre les intérêts de la chercheuse, ceux des participantes ainsi que le respect de la démarche scientifique pour toutes les étapes du projet. 


\section{$\&$}

\section{REVUE HYBRIDE DE L'ÉDUCATION}

\section{Outils de collecte de données}

Dans ce projet de recherche, plusieurs outils de collecte de données ont été utilisés. Une entrevue semi-dirigée a été réalisée avec chacune des enseignantes pour comprendre et expliciter leurs perspectives individuelles (Savoie-Zajc, 2009). Cette entrevue permettait de dresser un portrait initial des classes afin d'élaborer conjointement avec les enseignantes un projet pouvant répondre à leur besoin et au contexte de leur classe. Chacune des rencontres réflexives (rencontres de planification conjointe/retours réflexifs) a été enregistrée et transcrite. Cette transcription a permis d'analyser les données. Durant les mises en pratique, la chercheuse était présente en classe et accompagnait les enseignantes dans leur pratique ; elles en avaient manifesté le besoin. Des notes d'observations ont été consignées dans le journal de bord de la chercheuse. Ce journal contenait aussi des notes sur la narration des événements, les étapes de la recherche collaborative, les difficultés rencontrées et les problèmes éthiques auxquels tout chercheur en recherche qualitative peut être confronté (Baribeau, 2005; Plante, 2005; Roy, 2009). Enfin, une seconde entrevue a été réalisée avec les enseignantes à la fin du projet pour dresser un bilan de la recherche et redonner la parole aux participantes.

\section{Résultats}

La mise en place de quatre coins de jeu symbolique enrichis de matériels écrits (restaurant, clinique médicale, construction chasse) élaborée avec la chercheuse aura permis de dégager cinq rôles tirés de la pratique de trois enseignantes : conteuse, guide, informatrice, médiatrice et décideuse. Nous décrirons quelques-unes des interventions liées à chacun de ces rôles.

Le rôle de conteuse survient plus fréquemment dans le thème de la chasse, comparativement aux trois autres thèmes. Les livres ajoutés dans l'aménagement sont écrits en atikamekw et une tente sert d'accessoire de jeu et de lieu de rassemblement, facilitant la lecture aux enfants, ce que confirme l'enseignante B. L'enseignante A se questionne durant le projet sur la manière d'introduire et de lire des livres en situation de jeu : « J'ai feuilleté (le livre) juste pour moi. C'était pour regarder ce que je pouvais faire avec ça » (Réflexif, Ens. A, 24/03/15).

Dans le rôle de guide, les enseignantes réalisent quelques interventions. À titre d'illustration, elles associent des mots aux images. Dans le thème du restaurant, elles utilisent le menu écrit en atikamekw. L'enseignante A raconte : « J'ai montré l'image. Puis, c'est là que l'enfant m'a dit le mot [...] tu veux manger du Pirew (perdrix) », tout en désignant le mot écrit à côté de l'image. Dans le thème de l'hôpital, les enseignantes utilisent les affiches du corps humain écrites en atikamekw. L'enseignante 


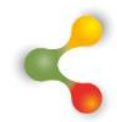

\section{REVUE HYBRIDE DE L'ÉDUCATION}

A explique : « J'ai demandé aux enfants dans quelle partie du corps ils ont mal. Puis j'ai demandé, c'est quoi ça ? Mais ils me les nomment en français, mais moi je les retraduis en atikamekw " (Réflexif, Ens. A, 17/02/15). Toujours dans le rôle de guide, les enseignantes gribouillent et écrivent des chiffres et des lettres devant les enfants en fonction de leur personnage. Par exemple, dans le thème de la construction, l'enseignante $C$ écrit un nombre sur un chèque de paye. Ce n'est que dans le thème de la chasse que les enseignantes écrivent des mots. Par exemple, l'enseignante B explique : "On a fait un plan pour partir à la chasse [...] j'ai écrit comme un plan » (Réflexif, Ens. B, 22/04/15). Dans celui-ci, deux mots sont écrits en atikamekw, ote et mos signifiant respectivement « lieu précisément ici » et un « orignal ».

Dans le rôle d'informatrice, les enseignantes expliquent la signification de mots de vocabulaire et la fonction des objets. Par exemple, avec le matériel de jeu ajouté dans le coin de la chasse, l'enseignante B se sert de la «puise et mentionne que c'est pour attraper les poissons » ou encore elle prend les jumelles et explique à un enfant qu'il peut «faire semblant de voir les animaux lorsqu'ils sont loin » (Mise en pratique, Ens, B., 21/04/15). Cette même stratégie s'emploie par l'enseignante C lorsque ce sont les enfants qui la questionnent sur la fonction du "sac de glace" ou du "guide des sentiers» (Mise en pratique, Ens. C, 20/04/15). Elle nomme le nom des objets et leur explique à quoi cela peut servir.

Dans le rôle de médiatrice, les enseignantes augmentent le temps de jeu. Avant le projet, l'enseignante A rapporte que les enfants ont le droit de jouer 15 minutes dans le coin. "C'est moi qui choisis qui s'en va jouer là. Le premier 15 minutes » (Planification, Ens. A, 18/03/15). Durant les mises en pratique accompagnées de la chercheuse, 45 minutes de jeu libre sont permises dans le coin de jeu symbolique, et ce, pour chacun des enfants.

Dans le rôle de décideuse, les enseignantes ont pris des décisions réfléchies et spontanées à l'égard du jeu et de l'émergence de l'écrit. Par exemple, elles ont choisi les thèmes, le matériel de jeu et le matériel écrit correspondant le mieux à leur contexte socioculturel.

\section{Discussion}

En présence de la chercheuse et avec des aménagements de coin de jeu symbolique enrichis de matériel écrit, les enseignantes atikamekws mettent en pratique cinq rôles en contexte de jeu symbolique pour favoriser l'émergence de l'écrit. Des particularités sont propres au contexte d'éducation préscolaire atikamekw dans lequel la recherche s'est déroulée. À titre d'illustration, certains rôles se sont réalisés spontanément durant les mises en pratique, par exemple, celui d'informatrice. En effet, les nouveaux thèmes de jeu choisis par les enseignantes (construction, clinique médicale, chasse) amènent les enfants à jouer avec du matériel de jeu 


\section{6}

\section{REVUE HYBRIDE DE L'ÉDUCATION}

dans la classe pour lequel ils ne sont pas familiarisés (ex. : puise, jumelle, thermomètre). Les enseignantes doivent alors expliquer la fonction de ces objets et faire découvrir de nouveaux mots de vocabulaire, parfois en atikamekw, et à d'autres occasions, en langue française. Les enseignantes possèdent un savoir issu de leur pratique qui se déploie dans des prises de décisions spontanées en contexte de jeu. À certaines occasions, la chercheuse suggère aux enseignantes des interventions pour favoriser l'émergence de l'écrit, notamment celle d'écrire des phrases et des mots en contexte de jeu plutôt que de gribouiller sur une feuille, un geste spontané en présence des enfants. Elles considèrent l'apprentissage de l'écrit, comme étant la capacité de reconnaître les "chiffres, les formes " (Initiale, Ens. A, 10/12/14) et les lettres de «l'alphabet» (Initiale, Ens. C, 16/03/15). Leurs interventions peuvent donc être influencées par leur croyance à l'égard de l'émergence de l'écrit (Sandvik, van Daal et Ade, 2014 ; Ure et Raban, 2001). De plus, bien qu'elles souhaitent faire découvrir l'écrit aux enfants, elles reconnaissent leur difficulté à traduire des mots en langue atikakemw, non seulement à l'écrit, mais aussi lorsqu'elles souhaitent raconter des histoires à partir des livres de lecture.

Les trois enseignantes ont aussi exprimé, à différents degrés, leurs découvertes en s'engageant dans le projet et leur trajectoire de leur implication. Nous proposons d'ouvrir la discussion d'abord sur leur développement professionnel, puis de discuter de la place de la collaboration de tous les acteurs, un enjeu fondamental pour mener à terme un projet de recherche en contexte autochtone.

\section{Réflexion sur le développement professionnel des enseignantes}

Les trois enseignantes sont unanimes sur le fait que le projet leur a permis de réfléchir à leurs rôles pour soutenir le jeu des enfants et favoriser l'émergence de l'écrit de ceux-ci, notamment en aménageant quatre coins de jeu symbolique dans leur classe. Toutes mentionnent qu'elles comprennent davantage l'importance de proposer aux enfants une variété de thèmes de jeu pour qu'ils puissent vivre des contextes ludiques d'apprentissage et développer leur langage oral et écrit. L'enseignante $A$ rapporte aussi sa plus grande présence à soutenir les enfants dans leur jeu : «: J'ai plus participé [...]. J'ai été plus présente. Pas juste dans les ateliers qu'on a montés, mais les autres aussi » (Bilan, Ens. A, 15/05/15). Les enseignantes se sentent aussi plus à l'aise de participer au jeu des enfants lorsque le thème est proche de leur culture autochtone ou immédiate, par exemple les thèmes de la chasse et du restaurant. Malgré ce fait, l'enseignante B souligne sa difficulté à s'ajuster au jeu et aux intérêts des enfants : "C'est juste que des fois, je savais pas quoi, comment on dit, quoi trop jouer». (Réflexif, Ens. B, 24/03/15). À la fin du projet, les enseignantes exposent les mêmes défis de temps et d'argent nécessaire pour planifier d'autres coins de jeu symbolique enrichis de matériels écrits. Une pratique réflexive sur la manière d'accompagner les enfants de 


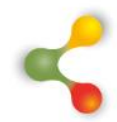

\section{REVUE HYBRIDE DE L'ÉDUCATION}

maternelle dans leur développement s'est donc amorcée par les enseignantes au cours de ce projet de recherche.

Des apprentissages liés à la langue atikamekw et à la langue française constituent un autre thème exprimé par les enseignantes. L'enseignante $C$ explique ses apprentissages liés aux deux langues : « J'ai un peu de la misère à parler en français, surtout les mots que j'entends, que je comprenais pas [...]. J'ai appris un peu sur la langue (française), sur ma langue (langue autochtone) » (Bilan, Ens. C, 15/05/15). L'enseignante $B$ qui possède une formation universitaire et six ans d'expérience en enseignement formule une autre explication :

J'ai appris que j'étais pas un dictionnaire. [...] Je suis encore en apprentissage tout le temps, parce que j'ai appris en français. J'arrive et on me dit d'enseigner la langue atikamekw, je n'ai pas toutes les connaissances qu'il faut [...] (Bilan, Ens. B, 13/05/15).

À ce sujet, cette enseignante utilise les services d'une technolinguiste lorsqu'elle rencontre des difficultés dans sa pratique.

Bien que le développement professionnel des enseignantes ne soit pas la visée première de la recherche collaborative (Bednarz, 2013; Desgagné et al., 2001), la démarche collaborative et réflexive dans laquelle les praticiennes se sont engagées aura permis des retombées concrètes dans leur pratique. À cet égard, leur implication, leur point de vue à toutes les étapes de la recherche collaborative et la préoccupation à tenir compte de leurs besoins ont joué un rôle dans leur propre développement professionnel (Uwamariya et Mukamurera, 2005).

\section{Réflexion sur la collaboration des acteurs concernés}

La mise en œuvre de ce projet de recherche réalisé avec trois enseignantes atikamekws a aussi impliqué d'autres acteurs clés, assurant son bon déroulement.

D'abord, le coordonnateur de l'éducation spécialisée a joué un rôle de pivot central en assurant le pont entre la chercheuse, les acteurs des services éducatifs et les membres du Conseil de bande. Son appui au projet et sa présence aux réunions administratives des services éducatifs ont facilité la conduite de la recherche. Dans plusieurs projets de recherche, les membres du Conseil de bande sont le point de chute naturel du projet (Asselin et Basile, 2012), alors que ce ne fut pas le cas dans notre situation.

Les membres du Conseil de bande, la direction de l'école et la direction des services éducatifs ont, quant à eux, été des acteurs dirigeants du projet, notamment en autorisant sa réalisation. Ils ont facilité sa mise en 


\section{6}

\section{REVUE HYBRIDE DE L'ÉDUCATION}

œuvre et contribué à sa pérennité en acceptant de payer la libération des enseignantes pour les rencontres et en acceptant d'héberger la chercheuse sans frais dans la communauté lors de ses déplacements. Ainsi, les défis de la collaboration entre les communautés autochtones et les chercheurs allochtones, tels que la distance géographique, la planification logistique et le coût des déplacements (Kish Anaquot Health Research [KAHR], 2008 ; Ritchie, Wabano, Beardy, Curran, Orkin, Vanderburgh et al., 2013) ont ainsi pu être simplifiés.

La technolinguiste de la communauté concernée a joué un rôle de transmetteur. Elle a apporté du soutien à l'équipe en traduisant ou encore en transmettant la norme orthographique de certains mots atikamekws. Son aide précieuse aura permis aux enseignantes une appropriation de la langue écrite atikamekw et à la chercheuse, la découverte de la complexité de cette langue.

Les parents et les enfants sont des acteurs périphériques à l'objet de cette recherche, mais qui se sont situés au cœur de cette aventure. Les enfants ont joué dans les coins de jeu mis en place par les enseignantes et la chercheuse. L'intérêt des parents a suscité un engouement et certains discutaient ouvertement du projet avec la chercheuse et les enseignantes.

Enfin, les trois enseignantes sont sans contredit les actrices principales du projet de recherche. La véritable collaboration entre cellesci et la chercheuse a permis à toutes et à chacune de partager leurs idées et leur expertise. Comme le souligne l'enseignante A : «On a beaucoup travaillé ensemble. [...] J'ai beaucoup aimé partager nos savoir-faire [...] » (Bilan, Ens. A, 15/05/15). Selon Gros-Louis McHugh (2012), la véritable collaboration ne se retrouve pas nécessairement dans l'approche méthodologique utilisée bien qu'elle doive être «personnalisée » (p. 5), la façon d'être en relation entre les différents acteurs impliqués permettra l'avancement du projet. La relation d'ouverture vis-à-vis les membres de la communauté concernée, l'expérience de travail et de recherche dans les milieux autochtones, mais surtout l'intérêt des trois enseignantes à connaître la chercheuse et à développer des liens d'amitié ont tour à tour contribué à créer et à maintenir une relation de confiance.

\section{Conclusion}

L'objectif de cet article était de discuter des rôles d'enseignantes en contexte de jeu symbolique pour favoriser l'émergence de l'écrit. Le savoir tiré de la pratique enseignante autochtone révèle que les trois participantes prennent des décisions à l'égard de l'émergence de l'écrit et du jeu de l'enfant dans leur contexte de classe. La dimension culturelle est un élément essentiel à tenir compte, notamment sur le thème de jeu et le matériel proposés aux enfants : les enseignantes ont alors plus de facilité à utiliser leur langue maternelle à l'oral et à l'écrit. 


\section{$\varepsilon$}

\section{REVUE HYBRIDE DE L'ÉDUCATION}

D'autres enjeux de cette recherche, dont le développement professionnel des enseignantes, ainsi que la collaboration des différents acteurs, ont trouvé un écho favorable. Selon un proverbe africain, il faut « tout un village pour élever un enfant». À la suite de l'expérience de cette recherche collaborative en milieu autochtone, il faut aussi la volonté et la collaboration de tous les membres d'une communauté pour assurer le déroulement et la pérennité d'un projet de recherche. Dans les contextes d'éducation préscolaire autochtone, l'utilisation de la recherche collaborative présente des forces méthodologiques qui contribuent à des retombées dans le milieu pratique. En plus de produire des connaissances scientifiques issues de la pratique ${ }^{2}$ et de répondre aux besoins des participantes, les enseignantes se sont engagées dans une démarche réflexive liée à leur pratique; elles ont été responsables de leur développement professionnel.

Les recherches en contexte d'éducation préscolaire en milieu autochtone sont encore peu nombreuses au Québec. II faudrait davantage de recherches collaboratives dans cette perspective où chercheurs et praticiens jumèlent leur expertise. L'esprit de collaboration entre les acteurs d'une communauté autochtone, les participants et les chercheurs est nécessaire pour tisser une culture de recherche collaborative répondant aux besoins réels des praticiens dans le respect des savoirs, de la langue et de la culture autochtone.

\footnotetext{
2 Des publications sont en cours de rédaction concernant d'autres résultats de la recherche. D'ici à ce que les articles soient publiés, tout lecteur est invité à prendre connaissance de la thèse doctorale de la chercheuse.
} 


\section{REVUE HYBRIDE DE L'ÉDUCATION}

\section{Références}

Asselin, H. et Basile, S. (2012). Éthique de la recherche avec les peuples autochtones, Éthique publique [En ligne], 14(1), récupéré le 15 décembre 2015 de http://ethiquepublique.revues.org/959.

Ball, J. (2007). Aboriginal young children's language and literacy development: Research evaluating progress, promising practices, and needs. Récupéré le 28 janvier 2013 du site Early Childhood Development Intercultural Partnerships, section "reports », http://www.ecdip.org/docs/pdf/CLLRNet\%20Feb\%202008.pdf.

Ball, J. (2009). Supporting young indigenous children's language development in Canada: a review of research on needs and promising practices. Canadian Modern Language Review, 66(1), 1947.

Ball, J. (2010). Promoting young indigenous children's emergent literacy in Canada. Récupéré le 28 janvier 2013 du site Early Childhood Development Intercultural Partnerships, section «reports", http://www.ecdip.org/docs/pdf/Emergent\%20literacy\%20Revised\%2 0Apr\%2027\%20811.pdf

Baribeau, C. (2005). L'instrumentation dans la collecte de données : le journal de bord du chercheur. Recherches qualitatives. Hors sérienuméro 2, 98-114.

Battiste, M. (2013). Docolonizing education : Nourishing the learning spirit. Saskatoon : Purish Publishing.

Battle, D. E. (2009). Multiculturalism, language and emergent literacy. Dans P. R. Rhyner (dir.), Emergent literacy and language development promoting learning in early childhood (p. 192-234). New York : The Guilford Press.

Bednarz, N. (2013). Regarder ensemble autrement: ancrage et développement des recherches collaboratives en éducation au Québec. Dans N. Bednarz (dir.). Recherche collaborative et pratique enseignante: regarder ensemble autrement (p. 13-29). Paris: L'Harmattan.

Bodova, E. et Leong, D. J. (2012). Les outils de la pensée: l'approche vygotskienne dans l'éducation à la petite enfance. Québec : Presses de l'Université du Québec.

Boisclair, A. et Sirois, P. (2010). Conclusion. Du préscolaire au primaire : avenues prospectives en recherche et intervention. Dans $\mathrm{H}$. Makdissi, A. Boisclair et P. Sirois (dir.), La littératie au préscolaire : 


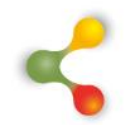

\section{REVUE HYBRIDE DE L'ÉDUCATION}

une fenêtre ouverte vers la scolarisation (p. 317-321). Québec: Presses de l'Université du Québec.

Boudreau, M. et Charron, A. (2014). Aménagement d'une aire de jeu symbolique et émergence de l'écrit. Revue pour la recherche en éducation, actes du colloque «Le jeu en contextes éducatifs pendant la petite enfance», Acfas 2013, 1-15.

Chilisa, B. (2012). Indigenous research methodologies. Thousand Oaks (USA) : SAGE Publications.

Commission de l'éducation (2007). Mandat d'initiative : la réussite scolaire des Autochtones. Récupéré le 26 novembre 2012 du site de l'Assemblée nationale, section «travaux parlementaires ", http://www.assnat.qc.ca.

Conseil en Éducation des Premières Nations [CEPN] (2002). La destinée de l'éducation pour les enfants des Premières Nations : Domaines prioritaires d'intervention. Récupéré le 29 octobre 2012 de http://www.cepn-

fnec.com/PDF/etudes documents/Rapport dossiers prioritaires.pd f.

Desgagné, S. (1997). Le concept de recherche collaborative : l'idée d'un rapprochement entre chercheurs universitaires et praticiens enseignants. Revue des sciences de l'éducation, 23(2), 371-393.

Desgagné, S. (2001). La recherche collaborative : nouvelle dynamique de recherche en éducation. Dans M. Anadón (dir.), Nouvelles dynamiques de recherche en éducation (p. 51-76). Québec: Les presses de l'Université Laval.

Desgagné, S., Bednarz, N., Lebuis, P., Poirier, L. et Couture, C. (2001). L'approche collaborative de recherche en éducation : un rapport nouveau à établir entre recherche et formation. Revue des sciences de l'éducation, 27(1), 33-64.

de Haan, D. (2005). Social pretend play: potentials and limitations for literacy development. European Early childhood Education Research Journal, 13(1), 41-55.

de la Sablonnière, R., Usborne E. et Taylor, D. M. (2011). Revivifier les langues autochtones meurtries: éliminer la discrimination systémique par l'enseignement. Dans L. Drapeau (dir.), Les langues autochtones du Québec: un patrimoine en danger (p.67-83). Québec : Presses de l'Université du Québec.

Dubet, F. (1994). La sociologie de l'expérience. Paris: Éditions du Seuil. 


\section{$\&$}

\section{REVUE HYBRIDE DE L'ÉDUCATION}

Eisazadeh, N., Rajendram, S., Portier, C. et Peterson, S. S. (2017). Indigenous Children's Use of Language During Play in Rural Northern Canadian Kindergarten Classrooms. Literacy Research: Theory, Method, and Practice, 66(1), 293-308.

Francis, N. et Reyhner, J.A. (2002). Language and literacy teaching for indigenous education: A bilingual approach. Clevedon, England: Multilingual Matters.

Giasson, J. (2011). La lecture : apprentissage et difficultés. Montréal : Gaëtan Morin.

Gillis, J. (1991). Play of first nations children in Ontario. Ottawa: Department of National Health and Welfare Canada.

Gmitrovà, V. et Gmitrov, J. (2003). The impact of teacher-directed and child-directed pretend play on cognitive competence in kindergarten children. Early Childhood Education Journal, 30(4), 241-246.

Gros-Louis McHugh, N. (2012). Aligner la recherche scientifique aux besoins et aux intérêts des premières nations: meilleures pratiques et initiatives prometteuses. Éthique publique [En ligne], 14(1), récupéré le 25 février 2016 de http://ethiquepublique.revues.org/961.

Hall, N. (2009). Literacy, play, and authentic experience. Dans K. A. Roskos et J. F. Christie (dir.), Play and literacy in early childhood : Research from multiple perspective ( $2^{\mathrm{e}}$ éd.) (p. 169-184). New York: Routledge.

Hot, A. (2010). Écrire et lire la langue inuit: choix linguistiques contemporains à lqaluit et lgloolik, Nunavut. Thèse de doctorat inédit, Université Laval.

Jacob, E., Charron, A. et da Silveira, Y. (2015a). Pretend play and emergent literacy for Aboriginal children. International Journal of Holistic Early Learning and Development, 1, 39-52.

Jacob, E, Charron, A et da Silveira, Y. (2015 b). Le jeu symbolique pour favoriser l'émergence de l'écrit des enfants autochtones en contexte de maternelle : possibilité et défis pour les enseignants autochtones. Revue de la persévérance et de la réussite scolaires chez les premiers peuples, $1,80-83$.

Jacob, E. (2013). La participation des mères atikamekws dans le jeu symbolique de leur enfant. Dans G. Maheux and R. Gauthier (Dir). La formation des enseignants inuits et des Premières Nations: 


\section{8}

\section{REVUE HYBRIDE DE L'ÉDUCATION}

structures, problématiques et pistes d'action (p. 9-30). Québec: Presses de l'Université du Québec.

Jacob, E. (2012). L'environnement de jeu de l'enfant atikamekw âgé de deux à quatre ans : la perspective des mères et leur implication dans le jeu symbolique de leur enfant, Mémoire de maitrise inédit, Université du Québec à Chicoutimi.

Jacob, E. (2017). Les rôles d'enseignantes atikamekws à l'éducation scolaire en contexte de jeu symbolique pour favoriser l'émergence de l'écrit : une recherche collaborative en milieu autochtone. Thèse de doctorat inédite. Université du Québec à Montréal.

Juster, A. H. et Leichter-Saxby, M. (2014). Citizens at Play: children's participation through community-based opportunities for childdirected play. Global Studies of Childhood, 4(2), 77-88.

Institut culturel éducatif montagnais [ICEM] (2007). Rapport d'activités : colloque sur la langue innue. Colloque «Uauitetau innu-aimun, aimuatamutau mamu » (Parlons de la langue innue, discutons-en ensemble). Sept-Îles, Québec.

Kish Anaquot Health Research [KAHR] (2008). La recherche collaborative : une perspective autochtone, traduit par Félix-Marie Affa'a, Ottawa: Coalition canadienne pour la recherche en santé mondiale.

Kovach, M. (2009). Indigenous methodologies - Characteristics, conversations, and contexts. Toronto : University of Toronto Press.

Larivée, S. J. (2011). Le rapport au savoir au préscolaire : impact sur la transition vers la $1^{\text {re }}$ année du primaire. Dans P. Maubant (dir.), Enjeux de la place des savoirs dans les pratiques éducatives en contexte scolaire. Compréhension de l'acte d'enseignement et défis pour la formation professionnelle des enseignants (p. 59-89). Québec : Presses de l'Université du Québec.

Larose, F. (1988). Le jeu traditionnel algonquin au sein du processus d'intégration socio-économique dans une société semi-nomade et son utilité pédagogique. Enfance, 41(3-4), 25-43.

Lavoie, C. (2016). Quelle est la meilleure démarche d'« amérindianisation » de la pratique d'enseignement ?. Revue Canada Education, 54, 2-2.

Lynch, J. (2015a). Preschool teachers' belief about the teaching and learning of language and literacy: Implications for education and practice. International Research in Early Childhood Education, 6(1), 91-111. 


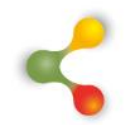

\section{REVUE HYBRIDE DE L'ÉDUCATION}

Lynch, M. (2015 b). More play, please : The perspective of kindergarten teachers on play in the classroom. American Journal of Play, 7(3), 347-370.

Ministère de l'Éducation du Québec [MEQ] (2001). Programme de formation de l'école québécoise. Éducation préscolaire. Enseignement primaire. Québec : Gouvernement du Québec.

Ministère de l'Éducation et de l'Enseignement supérieur [MEES] (2017). Programme de formation de l'école québécoise. Éducation préscolaire 4 ans. Québec : Gouvernement du Québec.

Montésinos-Gelet, I., Dupin de Saint-André, M. et Bourdeau, R. (2015). Accompagner des élèves en difficulté dans leur appropriation de l'écrit à l'aide de la littérature jeunesse. Revue de recherches en littératie médiatique miultimodale, 2, 1-51.

Neuman, S. B. et Roskos, K. (1993). Access to print for children of poverty : Differential effects of adult mediation and literacy-enriched play settings on environmental and functional print tasks. American Educational Research Journal, 30(1), 95-122.

Norris, M. (2015). L'évaluation du développement lexical chez les jeunes Innus : l'importance d'assurer un suivi en deux langues. Revue de la persévérance et de la réussite scolaires chez les Premiers Peuples, 1, 84-87.

Norris, M. (2007). Langues autochtones au Canada : nouvelles tendances et perspectives sur l'acquisition d'une langue seconde. Tendances sociales canadiennes, Produit no 11-008 au catalogue de Statistique Canada. Récupéré le 15 décembre 2012 de http://www.statcan.gc.ca/pub/11-008-x/2007001/9628-fra.htm.

Pellegrini, A. D. (1980). The relationship between kindergartners' play and achievement in prereading, language, and writing. Psychology in the Schools, 17(4), 530-535.

Peterson, S. S., Madsen, A., San Miguel, J., Jang, S. Y. (2018). Children's rough and tumble play: perspectives of teachers in northern Canadian Indigenous communities. Early Years, 38(1), 53-67.

Plante, B. (2005). Cheminement éthique d'un chercheur engagé en recherche collaborative. Revue des sciences de l'éducation, 31(2), 417-440.

Proulx, J. (2013). Réflexions épistémologiques sur la recherche collaborative en didactique : possibilités et excès. Dans N. Bednarz 


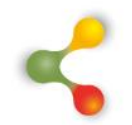

\section{REVUE HYBRIDE DE L'ÉDUCATION}

(dir.), Recherche collaborative et pratique enseignante: regarder ensemble autrement (p. 327-349). Paris : L'Harmattan.

Ritchie, S. D., Wabano, M. J., Beardy, J., Curran, J., Orkin, A., Vanderburgh, D. et Young, N. L. (2013). Community-based participatory research with Indigenous communities : The proximity paradox. Health and Place, 24, 183-189.

Roskos, K. et Neuman, S. B. (1993). Descriptive obsevations of adult' facilitation of literacy in young chldren's play. Early Childhood Research Quarterly, 8, 77-97.

Roy, S. N. (2009). L'étude de cas. Dans B. Gauthier (dir.), Recherche sociale: de la problématique à la collecte de données (5e éd.) (p. 199-225). Québec : Presses de l'Université du Québec.

Sandvik, J. M., van Daal, V. HP. et Adèr, H. J. (2014). Emergent literacy:Preschool teachers'beliefs and practices. Journal of Early Childhood Literacy, 14(1), 28-52.

Saracho, O. N. (2002). Teachers' roles in promoting literacy in the context of play. Early Child Development and Care, 172(1), 23-34.

Savoie-Zajc, L. (2009). L'entrevue semi-dirigée. Dans B. Gauthier (dir.) : Recherche sociale : de la problématique à la collecte de données $\left(5^{\mathrm{e}}\right.$ éd.). Québec, Québec : Presses de l'Université du Québec.

Sawyer, K. R. et DeZutter, S. (2009). Improvisation : A lens for play and literacy research. Dans K. A Roskos. et J. F. Christie (dir.), Play and literacy in early childhood: Research from multiple perspective $\left(2^{\mathrm{e}}\right.$ éd.) (p. 21-36). New York : Routledge.

Smith, L.T. (2012). Decolonizing Methodologies : Research and Indigenous Peoples ( $2^{\mathrm{e}}$ éd.). Londre: University of Otago Press.

Thériault, J. et Lavoie, N. (2004). L'éveil à la lecture et à l'écriture... Une responsabilité familiale et communautaire. Outremont, Québec: Éditions Logiques.

Toulouse, P.R. (2008). Integrating aboriginal teaching and values into the classroom. What Works ? Research into Practices. Récupéré du site Ontario Ministry of Education le 15 février 2016 de http://www.edu.gov.on.ca/eng/literacynumeracy/inspire/research/to ulouse.pdf

Ure, C.et Raban, B. (2001). Teachers' beliefs and understandings of literacy in the pre-school : Pre-school literacy project stage 1. Contemporary Issues in Early Childhood, 2(2), 157-158. 


\section{REVUE HYBRIDE DE L'ÉDUCATION}

Vygotski, L. (1967). Play and its role in the mental development of the child. Soviet Psychology, 5, 6-18.

Weber-Pillwax, C. (2001). What is Indigenous research? Canadian Journal of Native Education, 25(2), 166-174.

Wilson, S. (2003). Progressing toward an indigenous research paradigm in Canada and Australia. Canadian Journal of Native Education, 27(2), 161-178.

Wilson, S. (2008). Research is ceremony : Indigenous research methods. Halifax, Canada : Fernwood Publishing.

Wilson, S. (2013). Using indigenist research to shape our future. Dans M. Grey, J. Coates, M. Yellow Bird et T. Hetherington (dir.), Decolonizing social work (p. 311-322). Farnham, Angleterre : Ashgate. 